\title{
NATURAL LIMITS FOR HARMONIC AND SUPERHARMONIC FUNCTIONS
}

\author{
BY
}

\author{
J. R. DIEDERICH
}

\begin{abstract}
In this paper it is shown that Fatou's theorem holds for superharmonic functions in certain Liapunov domains if mean continuous limits are used in place of nontangential limits for which Fatou's theorem fails. Also, existence of mean continuous limits is established for certain semi-linear elliptic equations in Liapunov domains.
\end{abstract}

0. Fatou's theorem for harmonic functions in the $N$-dimensional unit ball guarantees the existence of nontangential, therefore radial, limits at almost every boundary point if the condition

$$
\sup _{0 \leq r<1} \int_{|y|=1}|u(r y)| d s(y)<\infty
$$

is satisfied; various generalizations can be found in $[\mathrm{Cl}],\left[\mathrm{Cr}_{1}\right],[\mathrm{HW}],[\mathrm{St}]$, $\left[W_{1}\right]$, and $\left[W_{2}\right]$. For $u(x)$ superharmonic and satisfying $(0.1)$ the "theorem" fails as Zygmund revealed by constructing a Green potential satisfying $(0.1)$ and failing to have a nontangential limit at any boundary point of the unit disc even though it still must have radial limits almost everywhere; see $\left[T_{1}\right]$ for the example and comments.

In $\left[D_{1}\right]$ we introduced the notion of mean continuous $(\mathrm{mc})$ limit, see $\S 1$ of this paper for the definition, which is stronger than nontangential limits for harmonic functions in Lipschitz domains, i.e., if $u(x)$ has an mc limit at $x_{0}^{\prime}$, then it has a nontangential limit at $x_{0}^{\prime}$. Even so, as we shall establish, Fatou's theorem holds for superharmonic functions using mc limits.

The nontangential case $\left({ }^{1}\right)$ has been pursued in $[\mathbf{A H}],[\mathrm{So}],\left[\mathrm{T}_{2}\right]$ and $\left[\mathbf{W}_{2}\right]$ for restricted classes of superharmonic functions with Widman showing in $\left[W_{2}\right]$ that the absolutely continuous density of a Green potential must satisfy a dimen-

Presented to the Society, January 23, 1975; received by the editors February 25, 1975 and, in revised form, October 22. 1975.

AMS (MOS) subject classifications (1970). Primary 31 B05, 31 B25, $35 \mathrm{~J} 25$.

Key words and phrases. Superharmonic, mean continuous limit, nontangential limit, radial limit, Liapunov domain, semilinear elliptic.

(1) See the end of $\$ 2$ for additional remarks on the nontangential case. 
Our results not only eliminate this condition for mc limits, but also yield as a corollary the radial limit theorem for potentials of absolutely continuous sionally dependent condition to guarantee nontangential limits. measures, and is considerably simpler than the proofs of radial limit theorems for superharmonic functions given in $\left[\mathbf{T}_{1}\right]$ and $[\mathrm{So}]$. However, radial limits for potentials of measures do not follow from our results.

In $\$ 2$ we establish some lemmas needed in $\$ 4$ and establish the mc limit theorem for superharmonic functions in certain Liapunov domains. In $\S 3$, harmonic functions in Lipschitz domains are examined to indicate limitations on mc limits. In $\$ 4$ we show that solutions of semilinear elliptic equations in Liapunov domains have mc limits and obtain the nontangential limits as a corollary. In $\$ 5$ we consider questions of uniqueness for harmonic and superharmonic functions and solutions of linear elliptic equations which assume their boundary values mean continuously.

We mention now our debt to the methods contained in $\left[\mathrm{Cr}_{1}\right]$ and $\left[\mathrm{W}_{2}\right]$ which we generously employ throughout.

Before proceeding, we note the following about the title of this paper: Both radial and mc limits exist for a natural class of superharmonic functions, while nontangential limits may fail to exist. On the other hand, if a superharmonic function has radial limit zero everywhere, it is not in general the Green potential of a measure, while it is if it has mc limit zero everywhere, see Theorem 6 , and hence the title.

1. We shall work in open, connected sets $\Omega$ in $R^{N}, 3 \leqslant N$, with $x=\left(x_{1}\right.$, $\left.\ldots, x_{N}\right)$. Let $|x-y|^{2}=\Sigma\left(x_{i}-y_{i}\right)^{2} ;|E|$, the measure of a set $E$ as dictated by the context; $\partial E$, the boundary of $E$, and $\bar{E}=E \cup \partial E$. For both volume and surface integrals $f_{E}$ will denote $|E|^{-1} \int_{E}$. Let $B(x, r)=\{y|| x-y \mid<r\}$; $\widetilde{B}(x, r)=B(x, r) \cap \Omega ;$ and $\bar{\partial} B(s, r)=B(x, r) \cap \partial \Omega ;$ when $\Omega=R_{+}^{N}=\{x \mid 0<$ $\left.x_{N}\right\}, x^{\prime}=\left(x_{1}, \ldots, x_{N-1}, 0\right)$. Constants depending on $N$ and $\Omega$ will be denoted simply by $k$ even though changing frequently; otherwise the dependence will be indicated by $k(\cdot)$. We use $d S(y)$ to indicate the natural surface area elements; $\omega_{N}$ is the surface area of the unit $N$-ball.

For $f(y)$ defined on $\partial \Omega$ and $u(x)$ in $L_{1}(\Omega)$, Lebesgue class, set

$$
u_{f}(y, r)=f_{\widetilde{B}(y, r)}|u(x)-f(y)| d x .
$$

We use the notation $u(y, r)$ when $f \equiv 0$. If

$$
\lim _{r \rightarrow 0} u_{f}\left(x_{0}^{\prime}, r\right)=0
$$


we say that $u(x)$ has the mc limit (mean continuous limit) $f\left(x_{0}^{\prime}\right)$ at $x_{0}^{\prime}$.

Several types of domains will be considered. Certain bounded domains $\Omega$ whose boundaries are locally given by $C^{1+\gamma}$ functions will be called Liapunov domains; see $\left[W_{2}\right]$ for specifics. See $\left[W_{3}\right]$ for the definition of Liapunov-Dini domains. Some Liapunov domains will be required to satisfy the additional smoothness condition,

There exists $0<\alpha$ so that for $y \in \partial \Omega$ there is a sphere of radius $\alpha$ tangent to $\Omega$ at $y$ and, except for $y$, interior to $\Omega$.

A bounded domain $\Omega$ whose boundary is given locally by functions which are Lipschitz continuous of order 1 will be called a Lipschitz domain. Such domains have the property that they are locally starlike; see [HW].

2. In this section, we establish the mc limit analogue of Fatou's theorem for harmonic and superharmonic functions in the unit ball and in Liapunov domains satisfying (1.3). Because the first four lemmas are needed in $\$ 4$, the proofs will be given for $R_{+}^{N}$ rather than for $B(0,1)$ since they are essentially the same.

Let $G(x, y)$ be the familiar Green function in $R_{+}^{N}$ for the Laplace operator.

LEMMA 1. If $h(x)$ is harmonic in $R_{+}^{N}$ and satisfies

$$
\sup _{0<x_{N}<1} \int_{\left|x^{\prime}\right|<1}\left|h\left(x_{1}, \ldots, x_{N}\right)\right| d x^{\prime}<\infty
$$

then $h(x)$ has me limits for almost every $\left|x_{0}^{\prime}\right| \leqslant 1$.

Proof. Let $\Omega_{1}$ be the set $\left\{x|| x^{\prime} \mid \leqslant 1,0<x_{N}<1\right\}$ and $D$ the subdomain for which $\left|x^{\prime}\right| \leqslant \rho$. By Green's representation formula and (2.1)

$$
\begin{aligned}
h(x)= & \omega_{N}^{-1} \int_{\bar{\partial} B(0, \rho)} G_{\nu} d \mu\left(y^{\prime}\right) \\
& +\omega_{N}^{-1} \int_{\partial D-\bar{\partial} B(0, \rho)} G_{\nu} h-G h_{\nu} d S(y)
\end{aligned}
$$

where $d \mu\left(y^{\prime}\right)=f\left(y^{\prime}\right) d y^{\prime}+d \psi\left(y^{\prime}\right)$ is the weak limit of $d \mu_{j}\left(y^{\prime}\right)=$ $h\left(y_{1}, \ldots, y_{N-1}, \tau_{j}\right) d y^{\prime}$ and subscript $\nu$ denotes outward normal derivatives; (2.1) gives $\int_{\Omega_{1}}|h| d y<\infty$ from which $\int_{\Omega_{1}} y_{N}\left|h_{y_{j}}\right| d y<\infty$ easily follows. Thus by Fubini's theorem and the familiar estimates $G(x, y) \leqslant$ $k x_{N} y_{N}|x-y|^{-N}$ and $\left|G_{\nu}(x, y)\right| \leqslant k x_{N}|x-y|^{-N}$, the existence of (2.3) is guaranteed for almost every $\rho \leqslant 1$; select and fix such a $\rho$.

Clearly (2.3) converges uniformly to zero for $\left|x_{0}^{\prime}\right|<\rho^{\prime}<\rho$; therefore let $h(x)=(2.2)$. Since almost every point $x_{0}^{\prime} \in \bar{\partial} B(0, \rho)$ is a Lebesgue point of 
$f\left(y^{\prime}\right) d y^{\prime}$ and $\psi\left(y^{\prime}\right)$ is singular, given $0<\epsilon$, there is $r_{0}=r_{0}\left(x_{0}^{\prime}\right)$ so that

$$
f_{\overline{\partial B}\left(x_{0}^{\prime}, r\right)} d \mu_{1}\left(y^{\prime}\right)<\epsilon, \quad 0<r \leqslant 2 r_{0} .
$$

$d \mu_{1}\left(y^{\prime}\right)=\left|f\left(y^{\prime}\right)-f\left(x_{0}^{\prime}\right)\right| d y^{\prime}+d|\psi|\left(y^{\prime}\right)$.

We need the following estimates:

$$
f_{\widetilde{B}\left(x_{0}^{\prime}, r\right)} G_{\nu}\left(x, y^{\prime}\right) d x \leqslant \begin{cases}k r^{1-N}, & \text { for } y^{\prime} \in \bar{\delta} B\left(x_{0}^{\prime}, 2 r\right), \\ k r\left|y^{\prime}-x_{0}^{\prime}\right|^{-N}, & \text { otherwise. }\end{cases}
$$

(2.4) follows since $\left|G_{\nu}\left(x, y^{\prime}\right)\right| \leqslant k\left|x-y^{\prime}\right|^{1-N}$ and so

$$
f_{\widetilde{B}\left(x_{0}^{\prime}, r\right)} G_{\nu}\left(x, y^{\prime}\right) d x \leqslant k f_{B\left(y^{\prime}, 4 r\right)}\left|x-y^{\prime}\right|^{1-N} d x \leqslant k r^{1-N} .
$$

(2.5) is immediate since $\left|G_{\nu}\left(x, y^{\prime}\right)\right| \leqslant k r\left|x-y^{\prime}\right|^{-N}$ and $\left|x_{0}^{\prime}-y^{\prime}\right| \leqslant 2\left|x-y^{\prime}\right|$ for $y \in \bar{\partial} B(0, \rho)-\bar{\partial} B\left(x_{0}^{\prime}, 2 r\right)$ and $x \in \widetilde{B}\left(x_{0}^{\prime}, r\right)$.

Using these estimates we have, recalling the notation of (1.1),

$$
\begin{aligned}
h_{f}\left(x_{0}^{\prime}, r\right) \leqslant k & f_{\bar{\partial} B\left(x_{0}^{\prime}, 2 r\right)} d \mu_{1}\left(y^{\prime}\right)+k \sum_{n=2}^{n_{0}} r \int_{\Delta_{n}(r)}\left|y^{\prime}-x_{0}^{\prime}\right|^{-N} d \mu_{1}\left(y^{\prime}\right) \\
& +k r r_{0}^{-N} \int_{\bar{\partial} B(0, \rho)-\bar{\partial} B\left(x_{0}^{\prime}, r_{0}\right)} d \mu_{1}\left(y^{\prime}\right)
\end{aligned}
$$

with

$$
\begin{aligned}
\Delta_{n}(r) & =\bar{\partial} B\left(x_{0}^{\prime}, 2^{n} r\right)-\bar{\partial} B\left(x_{0}^{\prime}, 2^{n-1} r\right), \quad 2^{n_{0}-1} r<r_{0} \leqslant 2^{n}{ }^{0} r, \\
& <k \sum_{n=1}^{n_{0}} 2^{-n} f_{\bar{\partial} B\left(x_{0}^{\prime}, 2^{n_{r}}\right)} d \mu_{1}\left(y^{\prime}\right)+k\left(r_{0}, x_{0}^{\prime}\right) r \\
& <k \epsilon+o(1) \text { as } r \rightarrow 0,
\end{aligned}
$$

and the lemma follows.

LEMMA 2. Let $\eta$ be a positive Borel measure in $R_{+}^{N}$ with $\int_{R_{+}^{N}} d \eta(y)<\infty$. For almost every $x_{0}^{\prime} \in \partial R_{+}^{N}$

$$
r f_{\widetilde{B}\left(x_{0}^{\prime}, r\right)} d \eta(y)=o(1) \text { as } r \rightarrow 0 .
$$

Proof. For $E \subset \partial R_{+}^{N}$ and $0 \leqslant \tau$, set $\Phi_{\tau}(E)=\int_{E_{\tau}} d \eta(y)$ where $E_{\tau}=$ $\left\{y \mid y^{\prime} \in E, 0<y_{N}<\tau\right\}$. $\Phi_{\tau}\left(\partial R_{+}^{N}\right)=\epsilon_{\tau}=o(1)$ as $\tau \rightarrow 0$. Let $d \Phi_{\tau} / d y^{\prime}=$ $g\left(y^{\prime}\right)$; then $\int_{\partial R_{+}^{N}} g\left(y^{\prime}\right) d y^{\prime} \leqslant \epsilon_{\tau}$ and thus $\left|K_{\tau}\right| \leqslant \sqrt{\epsilon_{\tau}}$ where $K_{\tau}=\left\{y^{\prime} \mid \sqrt{\epsilon_{\tau}}<\right.$ $\left.g\left(y^{\prime}\right)\right\}$. 
For $x_{0}^{\prime} \in \partial R_{+}^{N}-K_{\tau}$

$$
\underset{r \rightarrow 0}{\lim \sup } r f_{\widetilde{B}\left(x_{0}^{\prime}, r\right)} d \eta(y) \leqslant \lim \sup _{r \rightarrow 0} k r^{1-N_{\Phi_{\tau}}}\left(\bar{\partial} B\left(x_{0}^{\prime}, r\right)\right) \leqslant k \sqrt{\epsilon_{\tau}} .
$$

Let $0<\gamma$ and select a sequence $\tau_{j} \rightarrow 0$ so that $\bigcup_{j} K_{\tau_{j}} \mid<\gamma$. Hence for $x_{0}^{\prime} \notin$ $U K_{\tau_{j}}$ by (2.7), (2.6) holds and the lemma follows.

LEMMA 3. Let $\psi$ be a positive Borel measure in $R_{+}^{N}$ with support in $\left\{y|| y^{\prime} \mid \leqslant 1,0 \leqslant y_{N} \leqslant 1\right\}$ which satisfies

$$
\int_{R_{+}^{N}} y_{N} d \psi(y)<\infty .
$$

Then the Green potential $g(x)=\int_{R_{+}^{N}} G(x, y) d \psi(y)$ has mc limit zero for almost every $x_{0}^{\prime}$ in $\partial R_{+}^{N}$.

Before proving this, we note the following: (1) Condition (2.8) is minimal in guaranteeing the existence of $g(x, r)$ and is standard, (2) dimensional hypotheses as (6.2.2) in $\left[W_{2}\right]$ for the nontangential theorems are not required, (3) the density need not be absolutely continuous, and (4) the lemma together with Lemmas 2 and 4 yields an elementary and the simplest proof of the radial limit theorem for Green potentials of absolutely continuous measures $\psi$ satisfying (2.8).

Proof. As in Lemma 1

$$
\begin{aligned}
g\left(x_{0}^{\prime}, r\right) \leqslant & \int_{\widetilde{B}\left(x_{0}^{\prime}, 2 r\right)} f_{\widetilde{B}\left(x_{0}^{\prime}, r\right)} G(x, y) d x d \psi(y) \\
& +\sum_{n=2}^{n_{0}} \int_{\Delta_{n}(r)} f_{\widetilde{B}\left(x_{0}^{\prime}, r\right)} G(x, y) d x d \psi(y) \\
& +\int_{R_{+}^{N}-\widetilde{B}\left(x_{0}^{\prime}, r_{0}\right)} f_{\tilde{B}_{\left(x_{0}^{\prime}, r\right)}} G(x, y) d x d \psi(y)
\end{aligned}
$$

with

$$
\Delta_{n}(r)=\widetilde{B}\left(x_{0}^{\prime}, 2^{n} r\right)-\widetilde{B}\left(x_{0}^{\prime}, 2^{n-1} r\right), \quad 2^{n_{0}-1} r<r_{0} \leqslant 2^{n_{0}} r,
$$

and $r \int_{\widetilde{B}\left(x_{0}^{\prime}, r\right)} y_{N} d \psi(y)<\epsilon$ for $r \leqslant 2 r_{0}\left(x_{0}^{\prime}\right)$ by Lemma 2. The estimates

$$
f_{\widetilde{B}\left(x_{0}^{\prime}, r\right)} G(x, y) d x \leqslant \begin{cases}k y_{N} r^{1-N}, & \text { for } y \in \widetilde{B}\left(x_{0}^{\prime}, 2 r\right), \\ k r y_{N}\left|y-x_{0}^{\prime}\right|^{-N}, & \text { otherwise, }\end{cases}
$$

follow as in Lemma 1 using $G(x, y) \leqslant k y_{N}|x-y|^{1-N}$ for (2.9) and $G(x, y) \leqslant$ $k r y_{N}\left|y-x_{0}^{\prime}\right|^{-N}$ with $x \in \widetilde{B}\left(x_{0}^{\prime}, r\right)$ and $y \notin \widetilde{B}\left(x_{0}^{\prime}, 2 r\right)$ for (2.10). Continuing the inequality 


$$
\begin{aligned}
g\left(x_{0}^{\prime}, r\right) \leqslant & k r f_{\widetilde{B}\left(x_{0}^{\prime}, 2 r\right)} y_{N} d \psi(y)+k \sum_{n=2}^{n_{0}} r f_{\Delta_{n}(r)}\left|y-x_{0}^{\prime}\right|^{-N_{y_{N}} d \psi(y)} \\
& +k r r_{0}^{-N} \int_{R_{+}^{N}} y_{N} d \psi(y) \\
\leqslant & k \sum_{n=1}^{n_{0}} 2^{-n}\left(2^{n} r\right) f_{\widetilde{B}\left(x_{0}^{\prime}, 2^{\left.n_{r}\right)}\right.} y_{N} d \psi(y)+o(1) \\
\leqslant & k \epsilon \epsilon+o(1) \text { as } r \rightarrow 0
\end{aligned}
$$

completing the proof.

LEMMA 4. The Green potential of an absolutely continuous measure satisfying (2.8) has radial limit zero at almost every $x_{0}^{\prime}$.

Proof. We will consider limits along vertical lines; obvious modification in this and Lemma 2 will give the result for interior straight line segments. For $0<r, \operatorname{set}\left(x_{1}, \ldots, x_{N-1}, r\right)=x_{r}$. Then for $g(x)=\int_{R_{+}^{N}} G(x, y) f(y) d y$

$$
g\left(x_{r}\right)=\omega_{n}^{-1} \int_{\partial B\left(x_{r}, \rho\right)} G_{\nu}^{\rho}\left(x_{r}, y\right) g(y) d S_{\rho}(y)+\int_{B\left(x_{r}, \rho\right)} G^{\rho}\left(x_{r}, y\right) f(y) d y
$$

which follows from the Riesz decomposition theorem [H, p. 116] for $0<\rho<$ $r / 2$ where $G^{\rho}(x, y)$ is the Green function for $B\left(x_{r}, \rho\right)$. So

$$
\rho^{N-1} g\left(x_{r}\right) \leqslant k \int_{\partial B\left(x_{r}, \rho\right)} g(y) d S_{\rho}(y)+k \rho^{N-1} \int_{0}^{\rho} t^{2-N} \int_{\partial B\left(x_{r}, t\right)} f(y) d S_{t}(y) d t
$$

which upon integrating by parts and noting that $1<2 y_{N} r^{-1}$

$$
\begin{aligned}
\leqslant & k \int_{\partial B\left(x_{r} \rho\right)} g(y) d S_{\rho}(y)+k \int_{B\left(x_{r}, r / 2\right)} y_{N} f(y) d y \\
& +k r^{N-2} \int_{0}^{r / 2} t^{1-N} \int_{B\left(x_{r}, t\right)} y_{N} f(y) d y d t \\
& +k \rho^{N-1} r^{-1} \lim _{t \rightarrow 0} t^{2-N} \int_{B\left(x_{r}, t\right)} y_{N} f(y) d y .
\end{aligned}
$$

Select $x_{r}^{\prime} \notin \bigcup_{\tau_{\tau_{j}}}$ of Lemma 2 ; then given $0<\epsilon$

$$
t^{1-N} \Phi_{\tau_{j_{0}}}\left(\bar{\partial} B\left(x_{r}^{\prime} t\right)\right) \leqslant k \sqrt{\epsilon_{\tau_{j_{0}}}} \leqslant k \epsilon
$$

for $\tau_{j_{0}}$ sufficiently small and $0<t \leqslant r$ also sufficiently small. Hence the last term above vanishes and the third term is majorized by $k r^{N-1} \epsilon$, so continuing the inequality and integrating $\rho$ between 0 and $r / 2$ we get 


$$
\begin{aligned}
g\left(x_{r}\right) & \leqslant k f_{\widetilde{B}\left(x_{r}^{\prime}, 2 r\right)} g(y) d y+k r f_{B\left(x_{r}^{\prime}, 2 r\right)} y_{N} f(y) d y+k \epsilon \\
& \leqslant k \epsilon+o(1) \text { as } r \rightarrow 0
\end{aligned}
$$

by Lemmas 2 and 3 and the lemma follows.

The analogue of Fatou's theorem is

THEOREM 1. If $u(x)$ is superharmonic in $B(0,1)$ and is nonnegative, or satisfies (0.1), then $u(x)$ has an mc limit for almost every $\left|x_{0}^{\prime}\right|=1$.

Proof. By the Riesz decomposition theorem $u(x)=h(x)+g(x)$ where $h(x)$ is nonnegative and harmonic and $g(x)=\int_{|y|<1} G(x, y) d \psi(y), \psi(y)$ a positive measure. Select $x_{0}$ so that $g\left(x_{0}\right)<\infty$. Thus

$$
k\left(x_{0}\right) \int_{|y|<1}(1-|y|) d \psi(y) \leqslant \int_{|y|<1} G\left(x_{0}, y\right) d \psi(y)<\infty
$$

and the theorem follows from Lemmas 1 and 3 which clearly hold for $B(0,1)$. The proof for condition (0.1) is essentially the same.

In the remainder of this section we consider superharmonic functions in various Liapunov domains. If $\Omega$ is a Liapunov-Dini domain and $G(x, y)$ is its Green function with respect to the Laplace operator, then from Theorem 2.3 $\left[W_{3}\right]$ we have, where $\delta(x)$ is the distance from $x$ to $\partial \Omega$,

$$
\begin{gathered}
G(x, y) \leqslant k \delta(x)|x-y|^{1-N}, \\
\left|\partial / \partial x_{i} G(x, y)\right| \leqslant\left\{\begin{array}{l}
k|x-y|^{1-N}, \\
k \delta(y)|x-y|^{-N},
\end{array}\right. \\
\left|\partial^{2} / \partial x_{i} \partial y_{i} G(x, y)\right| \leqslant k|x-y|^{-N} .
\end{gathered}
$$

We also note that for $k_{N}\left\{|x-y|^{2-N}-|r x /| x|-y| x|/ r|^{2-N}\right\}=G_{r}(x, y)$, the Green function for the sphere of radius $r$ centered at the origin, that for $|x|$, $|y| \leqslant r / 2$

$$
k|x-y|^{2-N} \leqslant G_{r}(x, y) .
$$

LEMMA 5. The Green potential of a positive measure $\psi$ satisfying

$$
\int_{\Omega} \delta(y) d \psi(y)<\infty
$$

in $\Omega$, a Liapunov domain, has mc limit zero for almost every point on $\partial \Omega .\left(^{2}\right)$

(2) See Theorem 6 for the statement of the converse. 
If $\psi$ is absolutely continuous, the Green potential has radial limit zero almost everywhere.

Proof. The essential part of the proof involves establishing

$$
f_{\widetilde{B}\left(x_{0}^{\prime}, r\right)} G(x, y) d x \leqslant \begin{cases}k \delta(y) r^{1-N}, & y \in \widetilde{B}\left(x_{0}^{\prime}, 32 r\right), \\ k r \delta(y)\left|x_{0}^{\prime}-y\right|^{-N}, & \text { otherwise. }\end{cases}
$$

Case 1. If $y \in \widetilde{B}\left(x_{0}^{\prime} 32 r\right)$, then by $(2.11), G(x, y) \leqslant k \delta(y)|x-y|^{1-N}$, which upon averaging over $\widetilde{B}(x, 64 r)$ gives (2.13).

Case 2. If $y \in \Omega-\widetilde{B}\left(x_{0}^{\prime}, 32 r\right)$, set $\left|x_{0}^{\prime}-y\right|=a$ and define $D_{a}^{-}=\Omega-$ $\widetilde{B}\left(x_{0}^{\prime}, a / 8\right)$. Let $D_{a}^{+}$be a Liapunov domain such that $\Omega \cup B\left(x_{0}^{\prime}, a / 4\right) \subset D_{a}^{+} \subset$ $\Omega \cup B\left(x_{0}^{\prime}, a / 2\right)$. For $0 \leqslant a \leqslant a_{0}, a_{0}$ sufficiently small, the $D_{a}^{+}$'s can be constructed to be uniformly Liapunov, using property $3^{\circ}$ for Liapunov domains; see $\left[\mathrm{W}_{2}\right]$. Let $G_{a}(x, z)$ be the Green function for $D_{a}^{+}$and set

$$
\beta(z)=f_{\widetilde{B}\left(x_{0}^{\prime}, r\right)} G(x, z) d x, \quad \beta_{a}(z)=f_{\widetilde{B}\left(x_{0}^{\prime}, r\right)} G_{a}(x, z) d x .
$$

Clearly $\beta(z)$ and $\beta_{a}(z)$ are harmonic in $D_{a}^{-}$and continuous in $\overline{D_{a}^{-}}$. Let $\partial^{\prime} D_{a}^{-}=$ $\left\{z|| z-x_{0}^{\prime} \mid=a / 8, z \in \partial D_{a}^{-}\right\}$. Since $\beta(z) \equiv 0$ on $\partial D_{a}^{-}-\partial^{\prime} D_{a}^{-}$

$$
\beta(z) \leqslant k r a^{-1} \beta_{a}(z), \quad z \in \partial D_{a}^{-}-\partial^{\prime} D_{a}^{-} .
$$

For $x \in \widetilde{B}\left(x_{0}^{\prime}, r\right)$ and $z \in \partial^{\prime} D_{a}^{-}, G(x, z) \leqslant k r|x-z|^{1-N}$ by (2.11) and hence

$$
\beta(z) \leqslant k r a^{1-N}, \quad z \in \partial^{\prime} D_{a}^{-}
$$

also $G_{a}(x, z)$ dominates the Green function for $B\left(x_{0}^{\prime}, a / 4\right)$ so by (2.12), $k|x-z|^{2-N} \leqslant G_{a}(x, z)$ which gives

$$
k a^{2-N} \leqslant \beta_{a}(z), \quad z \in \partial^{\prime} D_{a}^{-},
$$

which by (2.16) gives

$$
\beta(z) \leqslant k r a^{-1} \beta_{a}(z), \quad z \in \partial^{\prime} D_{a}^{-},
$$

which by (2.15) and the maximum principle yields

$$
\beta(y) \leqslant k r a^{-1} \beta_{a}(y) \text {. }
$$

If $\delta(y) \leqslant a / 4$ then $\delta(y)=\operatorname{dist}\left(y, D_{a}^{+}\right)$and by $(2.11), G_{a}(x, y) \leqslant k_{a} \delta(y)$. $|x-y|^{1-N}$ so that $\beta_{a}(y) \leqslant k_{a} \delta(y)\left|x_{0}^{\prime}-y\right|^{1-N}$ and by $(2.17), \beta(y) \leqslant$ $k_{a} r \delta(y)\left|x_{0}^{\prime}-y\right|^{-N}$, which gives (2.14) since $k_{a}$ may be replaced by $k$ given the uniformity of the $D_{a}^{+}$'s. If $a / 4 \leqslant \delta(y)$, then $G(x, y) \leqslant k r\left|x_{0}^{\prime}-y\right|^{1-N} \leqslant$ 
$k r \delta(y)\left|x_{0}^{\prime}-y\right|^{-N}$, again giving (2.14). If $a_{0}<\left|x_{0}^{\prime}-y\right|$, the same argument holds by setting $a=a_{0}$.

The extension of Lemma 2 to Liapunov domains is straightforward and therefore assumed. With this and (2.13)-(2.14), the lemma follows just as in Lemma 3 and the radial limits follow from Lemma 4.

LEMma 6. If $u(x)=\int_{\partial \Omega} G_{\nu}(x, y) d \mu(y)$ in $\Omega$, a Liapunov-Dini domain, $\mu$ a measure of bounded variation on $\partial \Omega$, then wherever $d \mu / d s$ exists $u$ has $m c$ limit $d \mu / d s$.

Proof. Use the method of Lemma 1 in conjunction with (2.11).

THEOREM 2. If $u(x)$ is a nonnegative superharmonic function in $\Omega, a$ Liapunov domain satisfying (1.3), then $u(x)$ has an mc limit at almost every point on $\partial \Omega$.

Proof. As in Theorem 1, we have $u(x)=h(x)+g(x), g(x)=$ $\int_{\Omega} G(x, y) d \psi(y)$ and $h(x)$ nonnegative. By Theorem $2.5\left[\mathrm{w}_{3}\right]$ and Lemma 6, $h(x)$ has mc limits almost everywhere on $\partial \Omega$. Using the existence of interior tangential spheres we easily get $k \delta(y) \leqslant G(x, y)$ for $x$ fixed and therefore Lemma 5 applies.

Remarks. Prior to acceptance of this note we were informed of the results in [SW] by the referee. In [SW] the authors handle the question of nontangential limits for positive superharmonic functions on Lipschitz domains by showing that $L^{p}, 1 \leqslant p<N /(N-2)$, integral averages over interior cones of height $\delta$ are $o(1)$ as $\delta \rightarrow 0$, except for a set of harmonic measure zero. Consequently behavior in a full interior neighborhood of a boundary point is not considered and, as we show in $\$ 3$, for Lipschitz domains, $L^{1}$ averages over such neighborhoods will not in general exist even for positive harmonic functions. In this sense then, results in [SW] are best possible for Lipschitz domains, though not for smooth Liapunov domains whose full interior neighborhoods are used as our results establish. It is clear of course that in the restricted case of bounded superharmonic functions our results follow as corollaries to those of [SW].

3. Before considering mc limits in general Liapunov domains in the following section, we briefly consider the case of the more general Lipschitz domains. In [HW] Hunt and Wheeden established, along with more general results, that bounded as well as nonnegative harmonic functions in Lipschitz domains have nontangential limits except for a boundary set of harmonic measure zero. An analogue for mc limits holds in the bounded case and follows as a corollary to their result; however, the analogue in the nonnegative case fails as we shall now show by constructing a nonnegative harmonic function in $\Omega \subset R^{2}$ which is not 
locally integrable about a set of boundary points $E$ of positive harmonic measure.

Let $E$ be a cantor set of positive measure in $[0,1]$ and $I_{j}=\left(a_{j}, b_{j}\right)$ the complementary intervals. Form $\bar{\Omega}$ by adjoining to $[0,1]^{2}$ the right triangular sets $T_{j}$ bounded by $\left[a_{j}, b_{j}\right]$ and the lines of slope -1 and 1 through $a_{j}$ and $b_{j}$ respectively.

The function $x_{1} x_{2}\left(x_{1}^{2}+x_{2}^{2}\right)^{-2}$ is harmonic for $\left(x_{1}, x_{2}\right) \neq(0,0)$ and nonnegative in the first quadrant; also, it is not in $L_{1}$. Let $h_{j}\left(x_{1}, x_{2}\right)$ be the corresponding function with singularity at the principal vertex of $T_{j}$ and nonnegative in the quadrant formed by $T_{j}$. Since $h_{j}\left(x_{1}, x_{2}\right)$ is bounded in $[0,1]^{2}$ and nonnegative in $T_{j}$, we can, by adding a positive constant $c_{j}$, take $h_{j}\left(x_{1}, x_{2}\right)$ to be positive in $\Omega$. By Harnack's theorem [H, p. 33], $h\left(x_{1}, x_{2}\right)=$ $\sum_{j=1}^{\infty} h_{j}\left(x_{1}, x_{2}\right) / 2^{j} h_{j}(1 / 2,1 / 2)$ is positive and harmonic in $\Omega$ and clearly not in $L_{1}$ in a neighborhood of points in $E$.

THEOREM 3. If $h(x)$ is harmonic and bounded in $\Omega$, a Lipschitz domain, then $h(x)$ has mc limits on $\partial \Omega$ except on a set of negligible size. $\left({ }^{3}\right)$

Proof. As in [HW] we can assume that $\Omega$ is starlike with respect to the origin and represent $h(x)=\int_{\partial \Omega} K(x, y) f(y) d \omega^{\circ}(y)$, where $\omega^{\circ}$ is the harmonic measure on $\partial \Omega$ with respect to the origin and $|f(y)| \leqslant M$, the bound for $h(x)$. Given $0<\epsilon$, by Lusin's theorem, there is a continuous function $g(y)$ on $\partial \Omega$ such that $g(y)=f(y)$ except on a set $E$ of harmonic measure less than $\epsilon$ and $|g(y)| \leqslant M$. Since $\Omega$ is a regular domain for the Dirichlet problem

$$
h_{f}\left(x_{0}^{\prime}, r\right) \leqslant 2 M f_{\widetilde{B}\left(x_{0}^{\prime}, r\right)} \omega_{E}(x) d x+o(1)
$$

as $r \rightarrow 0$ for $x_{0}^{\prime} \in \partial \Omega-E$, where $\omega_{E}(x)=\int_{E} K(x, y) d \omega^{\circ}(y)$ is harmonic in $\Omega$ and from [HW] has radial limit zero almost everywhere $\omega^{\circ}$ on $\partial \Omega-E$. By Egoroff's theorem, we can assure that $\omega_{E}(x)$ has uniform radial limit zero on $\partial \Omega-E_{1}$ and $E_{1}$ has harmonic measure less than $2 \epsilon$. Then

$$
f_{\widetilde{B}\left(x_{0}^{\prime}, r\right)} \omega_{E}(x) d x \leqslant k r^{-N}\left\{\int_{\widetilde{B}\left(x_{0}^{\prime}, r\right) \cap \widetilde{E}_{1}}+\int_{\widetilde{B}\left(x_{0}^{\prime}, r\right) \cap \widetilde{E}_{1}^{c}}\right\} \omega_{E}(x) d x
$$

where

$$
\begin{aligned}
\widetilde{E}_{1} & =\left\{x: x=\rho y, 0 \leqslant \rho<1, y \in E_{1}\right\} \\
& \leqslant k r^{-N}\left|\widetilde{B}\left(x_{0}^{\prime}, r\right) \cap \widetilde{E}_{1}\right|+o(1) \quad \text { as } r \rightarrow 0 \\
& \leqslant k r^{1-N}\left|\bar{\partial} B\left(x_{0}^{\prime}, r\right) \cap E_{1}\right|_{H}+o(1)
\end{aligned}
$$

$\left({ }^{3}\right)$ The exceptional set is the union of two sets of harmonic measure and $(N-1)$ dimensional measure zero, respectively. I wish to thank Professor William P. Ziemer who pointed out that the relationship between these measures for Lipschitz domains is unknown. 
with ||$_{H}$ denoting the $(N-1)$-dimensional Hausdorff measure,

$$
\leqslant o(1) \text { as } r \rightarrow 0
$$

for all $x_{0}^{\prime} \in \partial \Omega-E_{1}$, except possibly a set $E_{2}$, with $\left|E_{2}\right|_{H}=0$, since $\Omega$ is a Lipschitz domain. Since $\epsilon$ was arbitrary, except for a set of harmonic measure and a set of $(N-1)$-dimensional Hausdorff measure zero, $h(x)$ has an mc limit.

The failure of mc limits for positive harmonic functions can be partially remedied by using a notion of approximate mc limits defined by replacing the set $\widetilde{B}(y, r)$ in $(1.1)$ by $\widetilde{D}(y, r)$ where (1) $\widetilde{D}(y, r)$ contains an interior cone with vertex at $y$, and (2) $\lim _{r \rightarrow 0}|\widetilde{D}(y, r)||\widetilde{B}(y, r)|^{-1}=1$. With this, analogues of the results of [HW] hold for approximate mc limits. This will not be pursued since the techniques basically involve reducing the problem to the bounded case of Theorem 3 by constructing subdomains as in [HW] in which the function is bounded.

4. In $\left[\mathbf{W}_{2}\right]$ Widman established the existence of nontangential limits for nonnegative harmonic functions in Liapunov domains by considering uniformly elliptic semilinear equations of the form

$$
a^{i j}(x) u_{i j}=F\left(x, u, u_{i}, u_{i j}\right)
$$

in $R_{+}^{N}$ since these are invariant under mappings between Liapunov domains. In this section, we intend to verify the mc limit analogue for solutions of (4.1) and get the existence nontangential limits as a corollary.

We assume the following about (4.1):

(i) $\left|a^{i j}(x)-a^{i j}(y)\right| \leqslant k|x-y|^{\alpha}, 0<\alpha, x \in \partial \Omega, y \in \bar{\Omega}$.

(ii) $\left|F\left(x, u, u_{i}, u_{i j}\right)\right| \leqslant k\left\{\delta^{-2}(x) \beta(\delta(x))+\delta^{\alpha-2}(x)|u|+\delta^{\alpha-1}(x)\left|u_{i}\right|+\right.$ $\left.\delta^{\alpha}(x)\left|u_{i j}\right|\right\}, \beta(t)$ nondecreasing and $\int_{0} \beta(t) / t d t<\infty$.

We say that $u(x)$ is a solution of (4.1) when (i) and (ii) are in force. If in addition $x \in \bar{\Omega}$ in (i) and $F$ is independent of $u_{i j}$ in (ii) we say that $u(x)$ is a solution of $(4.1)^{\prime}$.

From $\left[\mathrm{W}_{2}\right]$ we need:

(iii) the functions $a^{i j}(x)$ on $\partial \Omega$ can be extended into $\Omega$ by $\vec{a}^{i j}$ in $C^{\infty}(\Omega)$, $C^{\alpha}(\bar{\Omega})$, equal to $a^{i j}$ on $\partial \Omega$, and $\left|\operatorname{grad} \bar{a}^{i j}\right| \leqslant k \delta^{\alpha-1}(y)$.

(iv) If $u(x)$ is a solution of (4.1) and satisfies (2.1) with $\Omega=R_{+}^{N}$, then

$$
\int_{\left|x^{\prime}\right| \leqslant 1,0 \leqslant x_{N} \leqslant 2} x_{N}^{\alpha-1}|u|+x_{N}^{\alpha}\left|u_{i}\right|+x_{N}^{\alpha+1}\left|u_{i j}\right| d x<\infty,
$$

see line 7.1.2 of $\left[\mathrm{W}_{2}\right]$.

Let $G(x, y)$ be the Green function in $R_{+}^{N}$ for $a^{i j}\left(x_{\partial}^{\prime}\right) \partial^{2} / \partial x_{i} \partial x_{j}, x_{0}^{\prime} \in \partial R_{+}^{N}$. Under the appropriate linear transformation $G(x, y)$ is transformed to the Green 
function of the Laplace operator and $\partial R_{+}^{N}$ to a linear hyperplane. Thus by uniform ellipticity for (4.1), $G(x, y)$ has estimates, independent of $x_{0}^{\prime}$,

$$
\begin{aligned}
G(x, y) & \leqslant\left\{\begin{array}{l}
k|x-y|^{2-N}, \\
k x_{N}|x-y|^{1-N} ; k y_{N}|x-y|^{1-N}, \\
k x_{N} y_{N}|x-y|^{-N},
\end{array}\right. \\
\left|G_{i}(x, y)\right| & \leqslant\left\{\begin{array}{l}
k|x-y|^{1-N}, \\
k x_{N}|x-y|^{-N},
\end{array}\right. \\
\left|G_{i j}(x, y)\right| & \leqslant\left\{\begin{array}{l}
k|x-y|^{-N}, \\
k x_{N}|x-y|^{-1-N}
\end{array}\right.
\end{aligned}
$$

Clearly then Lemmas 3 and 4 hold for $G$ potentials as well as Lemma 1 for solutions of $a^{i j}\left(x_{0}^{\prime}\right) u_{i j}=0$.

LEMMA 7. If $u(x)$ is a solution of (4.1) in $R_{+}^{N}$ and satisfies (2.1), then $u(x)$ has an mc limit almost everywhere in $\left|x_{0}^{\prime}\right|<1$.

REMARKS. Although the proof follows that of Theorem $7.1\left[\mathrm{~W}_{2}\right]$, it is simpler since lemmas and arguments involving cones are eliminated. Radial limits follow as a corollary using Lemma 4.

Proof. Let $D_{\tau}=\left\{x\left|\tau<x_{N}<\tau+1,\right| x^{\prime} \mid<\rho\right\}, 0 \leqslant \tau$ and $\partial^{\prime} D_{\tau}$ the part of $\partial D_{\tau}$ satisfying $x_{N}=\tau$. Let $G^{\tau}(x, y)$ denote the Green function for $\tau<x_{N}$ and the operator $a^{i j}\left(x_{0}^{\prime}\right) \partial^{2} / \partial x_{i} \partial x_{j}$, and set $x_{\tau}=x+(0, \ldots, \tau) ; D_{0}=D$ and $G^{\circ}$ $=G$. $\rho$ will be selected and fixed in step (2) below.

By the standard Green formula representation we have

$$
\begin{aligned}
\omega_{N} u\left(x_{\tau}\right)= & \int_{\partial^{\prime} D_{\tau}} G_{\nu}^{\tau} u d S(y)+\int_{\partial D_{\tau}-\partial^{\prime} D_{\tau}} G_{\nu}^{\tau} u-G^{\tau} u_{\nu} d S(y) \\
& +\int_{D_{\tau}} G^{\tau}\left\{F+\left[\bar{a}^{i j}(y)-a^{i j}(y)\right] u_{i j}\right\} d y \\
& +\int_{B_{\sigma \tau}}+\int_{D_{\tau}-B_{\sigma \tau}} G^{\tau}\left[\bar{a}^{i j}\left(x_{0}^{\prime}\right)-\bar{a}^{i j}(y)\right] u_{i j} d y \\
= & I_{1}\left(x_{\tau}\right)+\cdots+I_{5}\left(x_{\tau}\right)
\end{aligned}
$$

with $v(y)=\left(\nu_{1}(y), \ldots, v_{N}(y)\right)$ the outward normal, $B_{\sigma \tau}=\left\{y|| x_{\tau}-y \mid \leqslant \sigma\right\}$, $x_{N} / 4 \leqslant \sigma \leqslant x_{N} / 2$. For appropriate $\tau_{j}, \lim _{\tau_{j} \rightarrow 0} \omega_{N} u\left(x_{\tau_{j}}\right)=\omega_{N} u(x)=$ $\lim _{\tau_{j} \rightarrow 0} I_{1}\left(x_{\tau_{j}}\right)+\cdots+I_{5}\left(x_{\tau_{j}}\right)=\widetilde{I}_{1}(x)+\cdots+\widetilde{I}_{5}(x)$ having mc limits $\omega_{N} f\left(x_{0}^{\prime}\right), 0, \ldots, 0$ respectively for a.e. $\left|x_{0}^{\prime}\right|<\rho$; we now demonstrate this.

(1) By (2.1), for some sequence $\tau_{j} \rightarrow 0, u\left(y_{1}, \ldots, y_{N-1}, \tau_{j}\right) d y^{\prime}$ conver- 
ges weakly to $d \bar{u}\left(y^{\prime}\right)=f\left(y^{\prime}\right) d y^{\prime}+d \psi\left(y^{\prime}\right)$ so

$$
\widetilde{I}_{1}(x)=\int_{\left|y^{\prime}\right|<\rho} G_{\nu}(x, y) f\left(y^{\prime}\right) d y^{\prime}+d \psi\left(y^{\prime}\right)
$$

which has mc limit $\omega_{N} f\left(x_{0}^{\prime}\right)$ for a.e. $\left|x_{0}^{\prime}\right|<\rho$. Note in this and the following steps that by (4.3) the dependence of $G(x, y)$ on $x_{0}^{\prime}$ can be neglected.

(2) By (4.2) and Fubini's theorem $\rho$ can be selected as close to 1 as desired and fixed so that

$$
\int_{\partial D-\partial^{\prime} D} y_{N}\left|u_{i}\right|+|u| d S(y)<\infty .
$$

With this and using the appropriate parts of (4.3) we have $\widetilde{I}_{2}(x)$ exists and has uniform limit zero for $\left|x_{0}^{\prime}\right| \leqslant \rho^{\prime}<\rho$.

(3) For $\left\{|F|+\left|\vec{a}^{i j}(y)-a^{i j}(y)\right|\left|u_{i j}\right|\right\} d y=d \psi(y)$ satisfies (2.8) using (4.2), (i), (ii), and (iii). Thus $\widetilde{I}_{3}(x)$ exists and has mc limit zero a.e. $\left|x_{0}^{\prime}\right|<\rho$ by Lemma 3.

(4) Clearly $\tilde{I}_{4}(x)$ exists for any $x_{N} / 4 \leqslant \sigma \leqslant x_{N} / 2$, the largest being given for $\sigma=x_{N} / 2$. So for $x \in \widetilde{B}\left(x_{0}^{\prime}, r\right)$,

$$
\begin{aligned}
\widetilde{I}_{4}(x) & \leqslant k r^{\alpha} \int_{B\left(x, x_{N} / 2\right)} G\left|u_{i j}\right| d y \\
& \leqslant k r^{\alpha} x_{N}^{-\alpha} \int_{B\left(x_{N}, 2\right)} y_{N}^{1+\alpha}|x-y|^{1-N}\left|u_{i j}\right| d y, \quad \text { since } x_{N} \leqslant 2 y_{N}, \\
& \leqslant k r^{\alpha} x_{N}^{-\alpha} \int_{\widetilde{B}\left(x_{0}^{\prime}, 2 r\right)} y_{N}^{1+\alpha}\left|u_{i j}\right||x-y|^{1-N} d y .
\end{aligned}
$$

So $f_{\widetilde{B}\left(x_{0}^{\prime}, r\right)}\left|\widetilde{I}_{4}(x)\right| d x \leqslant k r f_{\widetilde{B}_{(}\left(x_{0}^{\prime}, 2 r\right)} y_{N}^{1+\alpha}\left|u_{i j}\right| d y$ which goes to zero for a.e. $\left|x_{\alpha}^{\prime}\right|<\rho$ by (4.2) and Lemma 2 .

(5) To calculate $\lim _{\tau_{j} \rightarrow 0} I_{5}\left(x_{\tau_{j}}\right)$ we first integrate $I_{5}\left(x_{\tau_{j}}\right)$ by parts twice using Green's identities and then let $\tau_{j} \rightarrow 0$ after which we integrate $x_{N} / 4 \leqslant \sigma$ $\leqslant x_{N} / 2$ and divide by $x_{N} / 4$ and get

$$
\begin{aligned}
\widetilde{I}_{5}(x)= & \int_{\left|y^{\prime}\right|<\rho} G_{j}[*]^{N j}\left(f\left(y^{\prime}\right) d y^{\prime}+d \psi\left(y^{\prime}\right)\right) \\
& +\int_{\partial D-\partial^{\prime} D}[*]^{i j} u G_{j} \nu_{i}-G u_{i} \nu_{j} d S(y) \\
& +4 x_{N}^{-1} \int_{x_{N} / 4}^{x} \int_{\partial B_{\sigma}}[*]^{i j}\left\{G_{j} u v_{i}-G u_{i} \nu_{j}\right\} d S_{\sigma}(y) d \sigma \\
& +4 x_{N}^{-1} \int_{x_{N} / 4}^{x_{N} / 2} \int_{D-B_{\sigma}}\left\{G_{j i}[*]^{i j} u+G_{j} \bar{a}_{i}^{i j} u-G a_{j}^{i j} u_{i}\right\} d y d \sigma \\
= & J_{1}(x)+\cdots+J_{4}(x),
\end{aligned}
$$


with $*=\bar{a}^{i j}\left(x_{0}^{\prime}\right)-\bar{a}^{i j}(y), G_{j}=\partial / \partial y_{j} G$; existence is easily checked using (4.2), (4.3), (4.4), and (iii).

(5a) With a very slight modification of Lemma 1 we have $J_{1}(x)$ having mc limit zero for a.e. $\left|x_{0}^{\prime}\right|<\rho ; J_{2}(x)$ has uniform limit zero for $\left|x_{0}^{\prime}\right|<\rho^{\prime}<\rho$ as in (2); $J_{3}(x)$ has $\mathrm{mc}$ limit zero a.e. by the method in (4); letting $J_{4}(x)=$ $J_{4}^{1}(x)+J_{4}^{2}(x)-J_{4}^{3}(x)$ in taking the terms of the integrand separately, we have $J_{4}^{3}(x)$ has mc limit zero as in (3).

(5b) $J_{4}^{1}(x)$ and $J_{4}^{2}(x)$ can be handled in the same way; as such

$$
\begin{gathered}
f_{\widetilde{B}\left(x_{0}^{\prime}, r\right)}\left|J_{4}^{1}(x)\right| d x \leqslant k f_{\widetilde{B}\left(x_{0}^{\prime}, r\right)} \int_{D-B\left(x, x_{N} / 4\right)}\left|G_{i j}(x, y)\right|\left|x_{0}^{\prime}-y\right|^{\alpha}|u| d y d x \\
\leqslant k \int_{\widetilde{B}\left(x_{0}^{\prime}, 2 r\right)}\left|x_{0}^{\prime}-y\right|^{\alpha}|u| f_{\widetilde{B}\left(x_{0}^{\prime}, r\right)}|x-y|^{-N} \chi(x, y) d x d y \\
+k \sum_{n=2}^{n_{0}} 2^{-n}\left(2^{n} r\right) f_{\Delta_{n}(r)} y_{N}^{\alpha-1}|u| d y+k\left(r_{0}\right) r
\end{gathered}
$$

where $\chi(x, y)=1$ if $x_{N} / 4 \leqslant|x-y|,=0$ otherwise again with $2^{n 0^{-1}} r<r_{0} \leqslant$ $2^{n}{ }^{0} r$ and $\Delta_{n}(r)=\widetilde{B}\left(x_{0}^{\prime}, 2^{n} r\right)-\widetilde{B}\left(x_{0}^{\prime}, 2^{n-1} r\right)$. By Lemma 2 for almost every $x_{0}^{\prime}$ the series is less than $k \in \Sigma_{n=2}^{n}{ }_{2} 2^{-n}$ for $r_{0}\left(x_{0}^{\prime}\right)$ sufficiently small. $\chi(x, y) \leqslant$ $\chi_{1}(x, y)$ where $x_{1}(x, y)=1$ for $y_{N} / 5 \leqslant|x-y|$, and $=0$ otherwise; thus the first term is majorized by

$$
\begin{aligned}
& k r^{\alpha} \cdot f_{\widetilde{B}\left(x_{0}^{\prime}, 2 r\right)}|u| \int_{B(y, 4 r)-B\left(y, y_{N} / 5\right)}|x-y|^{-N} d x d y \\
& \quad \leqslant k r f_{\widetilde{B}\left(x_{0}^{\prime}, 2 r\right)} y_{N}^{\alpha-1}\left|\log y_{N}\right||u| d y=o(1) \quad \text { as } r \rightarrow 0
\end{aligned}
$$

for a.e. $\left|x_{0}^{\prime}\right|<\rho$ by Lemma 2. By (1)-(5b) the lemma is established for $\mathrm{mc}$ limits.

By the mapping in Theorem 7.3, Theorem 5.1 of $\left[\mathrm{W}_{2}\right]$, and our Lemmas 4 and 6 we have the following:

THEOREM 4. If $u(x)$ is a positive solution of (4.1)' in $\Omega$, a Liapunov domain, then $u(x)$ has an $m c$ limit, consequently radial limit, almost everywhere on $\partial \Omega$.

Lemma 8. If $u(x)$ is a solution of (4.1) in $\Omega$ and has $m c$ limit $f\left(x_{0}^{\prime}\right)$, then $u(x)$ has nontangential limit $f\left(x_{0}^{\prime}\right)$ at $x_{0}^{\prime}$.

Proof. Let $V_{x_{0}^{\prime}}$ be a cone with vertex at $x_{0}^{\prime}$ and fixed aperture $\alpha, \alpha<$ $\pi / 2$. For $y \in V_{x_{0}^{\prime}},\left|y-x_{0}^{\prime}\right| \leqslant k_{\alpha} \delta(y), 0<k_{\alpha}$. By Lemma $3.7\left[\mathrm{~W}_{2}\right]$ using the techniques of Theorem $4.1\left[\mathrm{~W}_{2}\right]$ we have for $1<p$, with $r=\delta(y) / 2$ 


$$
\begin{aligned}
\int_{B(y, r / 3)} \delta^{N p-N}(x)\left\{|u|^{p}+\delta^{p}\left|u_{i}\right|^{p}+\delta^{2 p}\left|u_{i j}\right|^{p}\right\} d x \\
\leqslant k\left[\int_{B(y, r)}|u| d x\right]^{p}+k \int_{B(y, r)} \delta^{2 p+N p-N}|f|^{p} d x .
\end{aligned}
$$

From the standard Green formula we get

$$
\begin{aligned}
|u(y)| \leqslant k r^{-N} \int_{B(y, r / 3)}|u(x)| d x+k r^{1-N} \int_{B(y, r / 3)}\left|u_{i}\right| d x \\
\quad+k \int_{B(y, r / 3)} G\left\{|F|+r^{\alpha}\left|u_{i j}\right|\right\} d x .
\end{aligned}
$$

But

$$
\begin{aligned}
r^{1-N} \int_{B(y, r / 3)}\left|u_{i}\right| d x \leqslant k r^{1-N} r^{N(p-1 / p)}\left\{\int_{B(y, r / 3)}\left|u_{i}\right|^{p} d y\right\}^{1 / p} \\
\leqslant k r^{-N}\left\{\int_{B(y, r / 3)} \delta^{N p-N+p}(x)\left|u_{i}\right|^{p}\right\}^{1 / p} \\
\leqslant k_{\alpha} f_{\widetilde{B}\left(x_{0}^{\prime}, k_{\alpha} r\right)}|u(x)| d x+r^{-N / p}\left\{\int_{B(y, r)} \beta^{p}(\delta(x)) d x\right\}^{1 / p} \\
=o(1) \text { as } y \rightarrow x_{0}^{\prime} .
\end{aligned}
$$

Next

$$
\begin{aligned}
r^{\alpha} \int_{B(y, r / 3)} G(y, x)\left|u_{i j}\right| d x \leqslant & k_{\alpha} r^{\alpha+N p-2-N}\left\{\int_{B(y, r / 3)}|x-y|^{(2-N) q}\right\}^{1 / q} \\
& \times\left\{\int_{B(y, r / 3)} \delta^{N p-N+2 p\left|u_{i j}\right|^{p}}\right\}^{1 / p}
\end{aligned}
$$

for $(N-2) q<N$

$$
\leqslant k_{\alpha^{\prime}} r^{\alpha-N} f_{B\left(x_{0}^{\prime}, k_{\alpha}^{r)}\right.}|u(x)| d x+o(1)=o(1) ;
$$

the remaining terms go to zero in a similar fashion.

5. Finally we consider the question of uniqueness. For classical solutions of uniformly elliptic linear equations

$$
a^{i j}(x) u_{i j}+b^{i}(x) u_{i}+c(x) u(x)=0 \quad(c(x) \leqslant 0)
$$

whose coefficients are $\alpha$-Hölder continuous in $\Omega$, a Liapunov domain, we have

THEOREM 5. If $u(x)$ is the solution of (5.1) in $\Omega$ and

$$
\begin{gathered}
u\left(x_{0}^{\prime}, r\right)=o(1) \text { for almost every } x_{0}^{\prime} \in \partial \Omega, \\
u\left(x_{0}^{\prime}, r\right)=O(1) \text { for every } x_{0}^{\prime} \in \partial \Omega,
\end{gathered}
$$

then $u(x) \equiv 0$. 
As in $\left[D_{2}\right]$ the proof will not depend on the existence theory for these equations; however, a proof using this theory and Serrin's extension of Harnack's inequality [Se] provides a similar result for Lipschitz domains, which we omit.

Proof. By Lemma $1\left[D_{2}\right]$,

$$
|u(x)| \leqslant k f_{B(x, \delta(x) / 2)}|u(y)| d y
$$

and thus by (5.2) has a radial limit zero almost everywhere. If we can show that $u(x)$ is bounded in $\Omega$, then by Theorem $7.5\left[\mathrm{~W}_{2}\right], u(x)$ converges uniformly to zero on $\partial \Omega$ and thus by the maximum principle $u(x) \equiv 0$. Clearly then, Lemma $3\left[D_{2}\right]$ holds for $\Omega$ and the remainder of the proof essentially follows the proof of Theorem $1\left[D_{2}\right]$.

For superharmonic functions we have

THEOREM 6. If $u(x)$ is superharmonic in $\Omega$, a Liapunov domain satisfying (1.3), and satisfies (5.2) and (5.3), then $u(x)$ is the Green potential of a unique measure $\psi$ satisfying the condition of Lemma 5 .

Proof. This follows easily using the techniques of Theorem $1\left[D_{1}\right]$, with Lemma 5 used in lieu of Theorem 1 [So], thereby giving an elementary proof of this result.

THEOREM 7. If $u(x)$ is harmonic in $\Omega$, a Liapunov-Dini domain, and satisfies (5.2) and (5.3), then $u(x) \equiv 0$.

Proof. The proof follows the lines of Theorem 6 with obvious modifications and simplifications due to the harmonicity of $u(x)$.

ADDED IN PROOF. There exists a finite valued function $f(y)$ defined on $|y|$ $=1$ and a harmonic function $u(x)$ such that $u_{f}\left(x_{0}^{\prime}, r\right)=o(r)$ as $r \rightarrow 0$ for all $\left|x_{0}^{\prime}\right|=1$ while $\int_{|y|=1}|f(y)| d y=\infty$; in other words, the uniqueness theorems hold for solutions in $L_{1}$ which do not necessarily satisfy (0.1). The example will be given in a subsequent paper.

\section{REFERENCES}

[AH] M. G. Arsov and A. Huber, On the existence of non-tangential limits of subharmonic functions, J. London Math. Soc. 42 (1967), 125-132. MR 34 \#2916.

[C1] A. P. Calderón, On the behaviour of harmonic functions at the boundary, Trans. Amer. Math. Soc. 68 (1950), 47-54. MR 11, 357.

[C $\left.\mathrm{C}_{1}\right] \mathrm{L}$. Carleson, On the existence of boundary values for harmonic functions in several variables, Ark. Mat. 4 (1962), 393-399. MR 28 \#2232.

$\left[\mathrm{C}_{2}\right] \longrightarrow$, Selected problems on exceptional sets, Van Nostrand, Princeton, N. J., 1967. MR 37 \#1576.

[D $D_{1}$ J. R. Diederich, Representation of superharmonic functions mean continuous at the boundary of the unit ball, Pacific J. Math. 54 (1974), 65-70. MR 50 \#13566. 
$\left[\mathrm{D}_{2}\right]$ J. R. Diederich, Application of Serrin's kernel parametrix to the uniqueness of $L_{1}$ solutions of elliptic equations in the unit ball, Proc. Amer. Math. Soc. 47 (1975), 341347. MR 50 \#7784.

[H] L. L. Helms, Introduction to potential theory, Wiley, New York, 1969. MR 41 \#5638.

[HW] R. A. Hunt and R. L. Wheeden, On the boundary values of harmonic functions, Trans. Amer. Math. Soc. 132 (1968), 307-322. MR 37 \#1634.

[L] J. E. Littlewood, On functions subharmonic in a circle. II, Proc. London Math. Soc. 28 (1928), 383-394. \#1420.

[R] W. Rudin, Real and complex analysis, McGraw-Hill, New York, 1966. MR 35

[Se] J. B. Serrin, Jr., On the Harnack inequality for linear elliptic equations, J. Analyse Math. 4 (1955/56), 292-308. MR 18, 398.

[So] E. D. Solomentcev, Sur les valeurs limites des functions sousharmoniques, Czechoslovak Math. J. 8 (83) (1958), 520-536. (Russian) MR 21 \#6483.

[St] E. M. Stein, On the theory of harmonic functions of several variables. II, Acta Math. 106 (1961), 137-174. MR 30 \#3234.

[SW] M. L. Silverstein and R. L. Wheeden, Superharmonic functions on Lipschitz domains, Studia Math. 39 (1971), 191-198. MR 47 \#3699.

[ $\mathrm{T}_{1}$ ] E. B. Tolsted, Limiting values of subharmonic functions, Proc. Amer. Math. Soc. 1 (1950), 636-647. MR 12, 609.

$\left[\mathrm{T}_{2}\right] \longrightarrow$ Non-tangential limits of subharmonic functions, Proc. London Math. Soc. (3) 7 (1957), 321-333. MR 20 \#1110.

[W 1 ] K.-O. Widman, On the boundary values of harmonic functions in $R^{3}$, Ark. Mat. 5 (1964), 221-230. MR 34 \#1544.

$\left[\mathrm{W}_{2}\right]-$ On the boundary behavior of solutions to a class of elliptic partial dfferential equations, Ark. Mat. 6 (1967), 485-533. MR 36 \#2949.

$\left[\mathrm{W}_{3}\right]$ - Inequalities for the Green function and boundary continuity of the gradient of solutions of elliptic differential equations, Math. Scand. 21 (1967), 17-37 (1968). MR 39 \#621.

DEPARTMENT OF MATHEMATICS, UNIVERSITY OF CALIFORNIA, DAVIS, CALIFORNIA 95616 